\title{
Panel of Reviewers
}

Reviewers are appointed by the Managing Editor to represent all elements of aviation technology and engineering scholarship and practice. An appointment to the Review Panel recognizes professional and academic achievement. Appointments are for a three-year term with the option of renewal. For membership consideration, submit a curriculum vita or industry resume to John H. Mott, Managing Editor.

\section{Reviewers for the current edition}

Troy Allen, Indiana State University

Thomas Q. Carney, Purdue University

Michael Dyrenfurth, Purdue University

Mel J. Futrell, Wright State University

Steven Hampton, Embry-Riddle Aeronautical University

Frederick Hansen, Oklahoma State University - Tulsa

Mary Johnson, Purdue University

Chien-tsung Lu, Purdue University

Eric Molin, Delft University of Technology

Fejda Netjasov, University of Belgrade

Samuel Pavel, Southern Illinois University - Carbondale

C. Daniel Prather, Middle Tennessee State University

José R. Ruiz, Southern Illinois University - Carbondale

Alan J. Stolzer, Embry-Riddle Aeronautical University

Roger Wayson, U. S. Department of Transportation

Jinn-Tsai Wong, National Chiao Tung University, Taiwan

Bernard Wulle, Purdue University 